\title{
Observation on Uncommon Complications of Acute Pancreatitis
}

\author{
Ram Dayal Singh ${ }^{1}$, Umbing Mudang2 ${ }^{2}$ Kumar Abhinav ${ }^{3}$ \\ ${ }^{1}$ Department of General Medicine, Patna Medical College, Patna, Bihar, India. ${ }^{2}$ Department of General Medicine, Patna \\ Medical College, Patna, Bihar, India. ${ }^{3}$ Department of General Medicine, Patna Medical College, Patna, Bihar, India.
}

\section{ABSTRACT}

\section{BACKGROUND}

Acute pancreatitis is a sudden inflammation of pancreas that lasts for a short time. It may range from mild discomfort to a severe, life threatening illness. Abdominal pain which is the major symptom of acute pancreatitis may be accompanied by nausea, vomiting and abdominal distension. ${ }^{1}$ There may be low grade fever with features of shock which is not unusual. ${ }^{2}$ Most people with acute pancreatitis recover completely after getting the right treatment. About $80 \%$ of cases of acute attack are self-limited and recover spontaneously within a week after the treatment. But in severe cases, acute pancreatitis can result in bleeding into the gland, serious tissue damage, infection and cyst formation. Severe pancreatitis can also harm other vital organs such as heart, lungs and kidneys. Transient hypotension, transient myocardial ischemia reflected by ST-T changes in ECG and associated with diastolic dysfunction and ARDS are uncommon complications of acute pancreatitis. This study was conducted to evaluate their incidence and prognostic significance in acute pancreatitis.

\section{METHODS}

This study was conducted in Patna Medical College Hospital, Patna, on admitted cases during the period august 2018 - august 2019. A total of 30 cases were included in this study who were diagnosed as acute pancreatitis and gave written consent for the same. Patients with association or history of stroke, head injury, CNS infection, features of demyelination, acute coronary syndrome, cardiogenic pulmonary oedema and established liver disease were excluded.

\section{RESULTS}

The incidence of transient hypotension, transient myocardial ischemia and ARDS in this study was found to be $13.3 \%, 6.6 \%$ and $6.6 \%$ respectively and they were all associated with high mortality.

\section{CONCLUSIONS}

The commonest risk factor of acute pancreatitis was seen to be Gall Stone followed by Alcoholism, hyperlipidaemia and smoking in this study also. The overall mortality $(13.3 \%)$ in this study was slightly lower than other studies. and this may be due to small sample size and variation of mortality with time of admission. Transient hypotension, transient myocardial ischemia reflected by ST-T changes in ECG, associated with diastolic dysfunction and ARDS were observed as uncommon complications of acute pancreatitis. Their incidence in this study were $13.3 \%, 6.6 \%$ and $6.6 \%$ respectively and they were all associated with high mortality. The clinical significance of their early detection is that the early mortality can be reduced with intensive medical and surgical management.

\section{KEY WORDS}

ARDS (Acute Respiratory Distress Syndrome), Transient Myocardial Ischemia, Transient Hypotension.

\author{
Corresponding Author: \\ Dr. Ram Dayal Singh, \\ Quarters No. 5, Black Harding Road, \\ Patna-800001, Bihar, India. \\ E-mail: umbing@gmail.com
}

DOI: $10.14260 / j e m d s / 2020 / 110$

Financial or Other Competing Interests: None.

How to Cite This Article:

Singh RD, Mudang $U$, Abhinav $K$. Observation on uncommon complications of acute pancreatitis. J. Evolution Med. Dent. Sci. 2020;9(08):488-491, DOI: 10.14260/jemds/2020/110

Submission 16-11-2019, Peer Review 28-01-2020, Acceptance 05-02-2020, Published 24-02-2020.

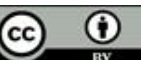




\section{BACKGROUND}

Acute pancreatitis is a sudden inflammation that lasts for a short time. It may range from mild discomfort to a severe, life threatening illness. Abdominal pain which is the major symptom of acute pancreatitis may be accompanied by nausea, vomiting and abdominal distension. There may be low grade fever with features of shock which is not unusual. ${ }^{2}$ Most people with acute pancreatitis recover completely after getting the right treatment. About $80 \%$ of cases of acute attack are self-limited and recover spontaneously within a week after the treatment. But in severe cases, acute pancreatitis can result in bleeding into the gland, serious tissue damage, infection and cyst formation. Severe pancreatitis can also harm other vital organs such as heart, lungs and kidneys.

Acute pancreatitis is a common disease with high mortality. ${ }^{3}$ Two most important risk factors are Gall stones and Alcohol ingestion. Other common causes can be hypertriglyceridemia and cigarette smoking. The risk of acute attack in patients is more even if there is one gall stone. Alcohol induced acute pancreatitis is more common in middle aged men. Pancreatitis evolves in three phases. Initial phase: where intrapancreatic digestive enzyme activation and acinar cell injury takes place. Second phase which involves activation, chemoattraction and sequestration of leucocytes and macrophages in the pancreas resulting in an enhanced intrapancreatic inflammatory reaction. The third phase is due to the effects of activated proteolytic enzymes and cytokines released by inflamed pancreas on distant organs.

Diagnosis is established by any two of the following criteria

1. Typical abdominal pain in the epigastrium which may be radiating to back.

2. Increase in threefold or more in serum lipase or amylase.

3. Cross sectional abdominal imaging which may have confirmatory findings. ${ }^{2}$

The two diagnostic biochemical markers elevated are the serum lipase and amylase. Amylase rises within hours and remains elevated for three to five days. Lipase is more specific but may be found elevated in other conditions also. But serum lipase is more preferred than amylase. However, there is no correlation between the severity of pancreatitis and the degree of serum lipase and amylase. Laboratory abnormalities encountered include hyperglycaemia, hypocalcaemia, leucocytosis, haemoconcentration, azotaemia, hypertriglyceridemia and mild elevations of liver function test results.

\section{Management}

Patient is kept NPO and given intravenous narcotic analgesics for pain and supplemental oxygen. Aggressive fluid resuscitation which is the most important intervention for acute attack is administered in the form of lactated Ringer's solution or normal saline. Initially bolus at $15-20 \mathrm{ml} / \mathrm{kg}$ followed by $2-3 \mathrm{ml} / \mathrm{kg} /$ hour to maintain urine output $>0.5$ $\mathrm{ml} / \mathrm{kg}$ /hour. Serial bedside evaluations done every 6-8 hours to assess vitals, oxygen saturation and change in physical examination to optimise fluid resuscitation. Measurement of haematocrit and BUN every 8-12 hours is recommended to ensure adequacy and response of fluid therapy. A decrease in haematocrit and BUN during first 12-24 hours is strong evidence for sufficient fluids. If it increases a volume challenge is done with $2 \mathrm{~L}$ crystalloid followed by increasing fluid rate by $1.5 \mathrm{ml} / \mathrm{kg} / \mathrm{hour}$. If it fails, ICU transfer is recommended for hemodynamic monitoring.

There is currently no role for prophylactic antibiotics for necrotising pancreatitis. Sterile necrosis is mostly managed conservatively but once diagnosis of infected necrosis is established pancreatic debridement should be considered. Symptomatic pseudocyst should be drained with surgery or endoscopy or by percutaneous route. For Pancreatic duct disruption after confirmation on MRCP or ERCP, a bridging pancreatic stent is placed for at least 6 weeks. Gallstone pancreatitis detected should be considered for cholecystectomy or endoscopic biliary sphincterotomy during admission or within 4-6 weeks of discharge. Patients with hypertriglyceridemia initial therapy include insulin, heparin or plasma pheresis. Alcoholic pancreatitis is also managed conservatively with abstinence from it on discharge. For outpatient, control of diabetes, lipid lowering agents and weight loss is advised. Nutritional therapy includes a low-fat solid diet for mild acute pancreatitis after abdominal pain has resolved. Enteral nutrition should be considered 2-3 days after admission for more severe pancreatitis instead of total parenteral nutrition. Enteral feeding maintains gut barrier integrity limits bacterial translocation and is less expensive with fewer complications than TPN. ${ }^{2}$ The mortality and morbidity associated with acute pancreatitis are the main concerns for a clinician. This disease is notable for complications leading to mortality and morbidity. Mild disease may resolve without serious complications in $80 \%$ cases $^{4}$ but remaining $20 \%$ cases with complication have mortality as high as $98 \%$. Hammel, et al, 5 categorised the disease into three-

1. Severe acute pancreatitis (with high mortality and morbidity). It is characterised by persistent organ failure which may be single or multiple for more than 48 hours.

2. Moderate acute pancreatitis (with high morbidity and low mortality). It is characterised by transient organ failure that may resolve within 48 hours.

3. Mild acute pancreatitis (with low morbidity and no mortality). ${ }^{6}$ There is no local complications or organ failure.

\section{Common Complications of Acute Pancreatitis}

1. Systemic- Inflammatory Response Syndrome (SIRS), Hypoxia, Hyperglycaemia, Hypocalcaemia, Hypoalbuminemia

2. Pancreatic- Necrosis of pancreatic cells, pancreatic abscess, Pseudocyst, Pancreatic Cysts, and Pleural effusion.

3. Gastrointestinal- Gastric or duodenal bleed, Variceal bleed, Erosion into colon, Duodenal obstruction (compression by pancreatic mass) and Obstructive Jaundice (CBD compression by swollen pancreas)

There are certain uncommon complications which are being reported recently and those are transient hypotension, ARDS and transient myocardial ischemia. In this study we have devoted ourselves to make an observation on these uncommon complications and their prognostic significance. 


\section{METHODS}

This is an observational study. Patients from in-patient male and female wards of Patna Medical College Hospital, Patna, admitted during August 2018- August 2019 were included in the study. A total of 30 cases were included in this study who were diagnosed as acute pancreatitis and gave written consent for the same. Patients with association or history of stroke, head injury, CNS infection, features of demyelination, acute coronary syndrome, cardiogenic pulmonary oedema and established liver disease were excluded.

\section{Diagnosis}

Diagnosis was made with the help of detailed history and clinical examination, routine investigations including Complete blood count, Routine examination of urine, Random blood sugar, Renal function test, Liver function test, Lipid profile along with special investigations including serum Amylase, Lipase, Lactate dehydrogenase, total and ionised calcium, USG of whole abdomen, CECT abdomen, Chest x-ray, Arterial blood gas analysis, ECG and echocardiography. Certain investigations like Culture of blood or Urine, Ascitic/pleural fluid study and CT brain were done as and when required

\section{Management}

Cases were managed using standard regiments and were continuously monitored till discharge, severity of disease was decided using Ranson's and Glasgow's scores. All cases were managed in in-patient department except two out of four who developed ARDS and needed ICU. Other cases with common complications (pancreatic cyst and pseudocyst) were also managed conservatively.

\section{RESULTS}

Total 30 cases were included in this study. Table 1 shows age and sex wise incident. We found that acute pancreatitis was found more common in males $(63.3 \%)$ than females $(36.7 \%)$ with male female ratio of 1.72:1

\begin{tabular}{|c|c|c|c|c|}
\hline & Male & Female \\
\hline Age Groups & No. of Patients & $\mathbf{\%}$ & No. of Patients & $\%$ \\
\hline $20-30$ & 04 & 13.3 & 03 & 10.0 \\
\hline $31-40$ & 05 & 16.7 & 02 & 6.7 \\
\hline $41-50$ & 09 & 30.0 & 05 & 16.7 \\
\hline $51-60$ & 01 & 3.3 & 01 & 3.3 \\
\hline Total & $\mathbf{1 9}$ & $\mathbf{6 3 . 3}$ & $\mathbf{1 1}$ & $\mathbf{3 6 . 7}$ \\
\hline \multicolumn{4}{|c}{ Table 1. Age and Sex Distribution $(\boldsymbol{n}=\mathbf{3 0})$} \\
\hline
\end{tabular}

\begin{tabular}{|c|c|c|}
\hline Comorbidities & No. of Patients & Percentage \\
\hline Gall stone & 22 & 73.3 \\
\hline Alcoholism & 15 & 50.0 \\
\hline Hyperlipidaemia & 12 & 40.0 \\
\hline Cigarette smoking & 09 & 30.0 \\
\hline Idiopathic & 04 & 13.3 \\
\hline \multicolumn{2}{|c|}{ Table 2. Risk Factors } \\
\hline
\end{tabular}

\begin{tabular}{|c|c|c|}
\hline & No. of Patients & Percentage \\
\hline Atelectasis & 1 & 3.3 \\
\hline Pleural effusion & 4 & 13.3 \\
\hline Pneumonia & 2 & 6.7 \\
\hline Bilateral lung opacity & 2 & 6.7 \\
\hline Normal & 23 & 76.6 \\
\hline Table 3. Chest X-Ray Findings of Acute Pancreatitis Patients \\
\hline
\end{tabular}

Table 3 X- ray shows Atelectasis, Pneumonia, Pleural effusion and bilateral opacity. Our concerns are cases showing bilateral opacities because these are the cases with ARDS.

\begin{tabular}{|c|c|c|}
\hline \multicolumn{3}{|c|}{ USG Finding of Acute Pancreatitis Patients } \\
\hline & No. of Patients & $\%$ \\
\hline Interstitial oedematous pancreatitis & 2 & 6.7 \\
\hline Pancreatic necrosis & 4 & 13.3 \\
\hline Pancreatic pseudocyst & 4 & 13.3 \\
\hline Increased pancreas volume & 20 & 66.7 \\
\hline \multicolumn{3}{|c|}{$\begin{array}{l}\text { CT Findings of Acute Pancreatitis } \\
\end{array}$} \\
\hline Focal and diffuse enlargement of the pancreas & 3 & 10.0 \\
\hline Pancreatic changes \& peripancreatic inflammation & 21 & 70 \\
\hline Acute pancreatic fluid collection & 2 & 6.7 \\
\hline Pancreatic necrosis & 4 & 13.3 \\
\hline \multicolumn{3}{|c|}{ ECG Findings of Acute Pancreatitis Patients } \\
\hline ST segment depression & 1 & 3.3 \\
\hline Diffuse $\mathrm{T}$ wave inversion & 1 & 3.3 \\
\hline Normal & 28 & 93.3 \\
\hline $\begin{array}{r}\text { Table 4. USG Findings, CT Findings and } \\
\text { Pancreatitis Patien }\end{array}$ & G Findings $o$ & \\
\hline
\end{tabular}

Table 4. USG is a chief tool for the diagnosis of Acute pancreatitis and also Pancreatic necrosis which was found in four cases who couldn't be saved. CT abdomen was also done in all cases and this table shows different CT findings In ECG findings two cases showed ST-T changes and one of them died

\begin{tabular}{|c|c|c|c|c|c|c|c|c|}
\hline \multirow{3}{*}{ Systolic } & \multicolumn{2}{|c|}{$<90 \mathrm{mmHg}$} & \multicolumn{2}{|c|}{$100-120 \mathrm{mmHg}$} & \multicolumn{4}{|c|}{$120-140 \mathrm{mmHg}>140 \mathrm{mmHg}$} \\
\hline & No & $\%$ & \begin{tabular}{|l|l} 
No \\
\end{tabular} & $\%$ & No & $\begin{array}{l}\% \\
\end{array}$ & $\mathrm{No}$ & \begin{tabular}{|l|l|} 
& $\%$ \\
\end{tabular} \\
\hline & 4 & 13.3 & 2 & 6.7 & 20 & 66.7 & 4 & 13.3 \\
\hline \multirow{3}{*}{ Diastolic } & \multicolumn{2}{|c|}{$<50 \mathrm{mmHg}$} & \multicolumn{2}{|c|}{$60-70 \mathrm{mmHg}$} & \multicolumn{2}{|c|}{$70-80 \mathrm{mmHg}$} & \multicolumn{2}{|c|}{$>80 \mathrm{mmHg}$} \\
\hline & No & $\%$ & \begin{tabular}{l|l} 
No \\
\end{tabular} & $\%$ & No & $\%$ & $\mathrm{No}$ & \begin{tabular}{l|l} 
& $\%$ \\
\end{tabular} \\
\hline & 4 & 13.3 & 2 & 6.7 & 20 & 66.7 & 4 & 13.3 \\
\hline \multicolumn{9}{|c|}{$\begin{array}{c}\text { Table 5. Systolic and Diastolic BP Measurement of Acute } \\
\text { Pancreatitis Patients }\end{array}$} \\
\hline \multicolumn{9}{|c|}{$\begin{array}{l}\text { There are two types of hypotension in Acute Pancreatitis- Transient and Persistent, } \\
\text { Four cases in this table had transient hypotension and two of them died. }\end{array}$} \\
\hline \multicolumn{4}{|c|}{ Parameters } & \multicolumn{2}{|c|}{ No. of Cases } & \multicolumn{2}{|c|}{ Discharge } & Death \\
\hline \multicolumn{4}{|c|}{ Acute pancreatitis with hypotension } & \multicolumn{2}{|c|}{04} & \multicolumn{2}{|c|}{02} & 02 \\
\hline \multicolumn{4}{|c|}{ Acute pancreatitis with ARDS } & \multicolumn{2}{|r|}{02} & \multicolumn{2}{|l|}{01} & 01 \\
\hline \multicolumn{4}{|c|}{$\begin{array}{l}\text { Acute pancreatitis with myocardial } \\
\text { dysfunction }\end{array}$} & \multicolumn{2}{|r|}{02} & \multicolumn{2}{|l|}{01} & 01 \\
\hline \multicolumn{4}{|c|}{ Acute pancreatitis } & \multicolumn{2}{|c|}{22} & \multicolumn{2}{|l|}{22} & 00 \\
\hline
\end{tabular}

\section{DISCUSSION}

In this prospective, non- randomised, uncontrolled observational study conducted in Patna Medical College Hospital Patna during August 2018 - August 2019, the overall early mortality was found to be $13.3 \%$ and it is slightly lower. This may be due to small sample size and variation in mortality with time of admission. ${ }^{7}$ All four cases who died were having pancreatic necrosis where surgical debridement is required, and this facility is not available in this institution. In this study four cases $(13.3 \%)$ show transient hypotension and four cases showed elevated blood pressure. Thandassery $\mathrm{R} \mathrm{B}$, et al ${ }^{6}$ reported transient hypotension in $19 \%$ cases and these cases were found to be associated with diastolic dysfunction. Again, small sample size may be the cause of slightly lower percentage of transient hypotension in this study but the mortality in this group was seen to be similar (50\%).

ST-T changes with diastolic dysfunction was seen in $6.6 \%$ cases. Pezilli, et $\mathrm{al}^{8}$ has reported ST-T changes in $51.8 \%$ cases. Here this study differs but figures of mortality (50\%) is similar to Pezilli study. Incidence of ARDS was found in $6.6 \%$ cases and 
mortality in this group also was $50 \%{ }^{9}$. Important facts the study reveals is that transient hypotension (13.3\%), transient myocardial ischemia (6.6\%) with ST-T changes and diastolic dysfunction and ARDS (6.6\%) are relatively uncommon complications of acute pancreatitis but they are associated with high early mortality ${ }^{10}$. Their early detection warrants intensive therapy and management including surgical debridement to reduce early mortality ${ }^{11}$. This is the important clinical significance of early detection of these uncommon complications. The basic pathology in all four cases who died was pancreatic necrosis ${ }^{12}$.

\section{CONCLUSIONS}

The commonest risk factor of acute pancreatitis was seen to be Gall Stone followed by Alcoholism, hyperlipidaemia and smoking in this study also. The overall mortality $(13.3 \%)$ in this study was slightly lower than other studies. and this may be due to small sample size and variation of mortality with time of admission. Transient hypotension, transient myocardial ischemia reflected by ST-T changes in ECG, associated with diastolic dysfunction and ARDS were observed as uncommon complications of acute pancreatitis. Their incidence in this study were $13.3 \%, 6.6 \%$ and $6.6 \%$ respectively and they were all associated with high mortality. The clinical significance of their early detection is that the early mortality can be reduced with intensive medical and surgical management.

\section{REFERENCES}

[1] Penman ID, Lees CW. Acute pancreatitis. Davidson Principles and practice of medicine. 22nd edn. 2014: p. 889-92.
[2] Conwell DL, Banks PA, Greenberger NJ. Acute and chronic pancreatitis. Harrison's Principles of Internal Medicine 20 th edn. McGraw-Hill Publication 2018;2:2437-44.

[3] Jovicic I, Petronijevic L, Denic L, et al. Epidemiology of acute pancreatitis in Belgrade. Pancreatology 2009;9:510.

[4] Lund $\mathrm{H}$, Tonnesen $\mathrm{H}$, Tonnesen $\mathrm{MH}$, et al. Long-term recurrence and death rates after acute pancreatitis. Scand J Gastroenterol 2006;41(2):234-8.

[5] Hammel P, Soufir N, Levy P, et al. Detection of CDKN2A, CDK and BRCA2 genes in patients with familial pancreatic cancer. Pancreatology 2009;9:463.

[6] Thandassery RB. Pancreas. 2017;46(5):625-30.

[7] Mutinga M, Rosenbluth A, Tenner SM, et al. Does mortality occur early or late in acute pancreatitis. Int J Pancreatol 2000;28(2):91-5.

[8] Pezilli R, Barakat B, Billi P, et al. Electrographic abnormalities in acute pancreatitis. Eur J Emerg Med 1999;6(1):27-9.

[9] Petrov MS, Windsor JA. Classification of the severity of acute pancreatitis: how many categories make sense? Am J Gastroenterol 2010;105(1):74-6.

[10] De Pretis N, Amodio A, Frulloni L. Hypertriglyceridemic pancreatitis: epidemiology, pathophysiology and clinical management. United European Gastroenterol J 2018;6(5):649-55.

[11] Johnstone C. Pathophysiology and nursing management of acute pancreatitis. Nurs Stand 2018 Jun 28.

[12] Gullo L, Migliori M, Olah A, et al. Acute pancreatitis in five European countries: etiology and mortality. Pancreas 2002;24(3):223-7. 